\title{
Amikacin resistance due to the aphA6 gene in multi-antibiotic resistant Acinetobacter baumannii isolates belonging to global clone 1 from Iran
}

\author{
Parisa Aris ${ }^{1}$, Mohammad Ali Boroumand ${ }^{2}$ and Masoumeh Douraghi ${ }^{1,3^{*}}$ (D)
}

\begin{abstract}
Background: TnaphA6-carrying repAci6 plasmids have been detected in Acinetobacter baumannii isolates belonging to global clones, GC1 and GC2, worldwide. Here, we examined whether RepAci6 plasmids family play a role in the dissemination of the aphA6 in GC1 A. baumannii isolates from Iran.

Results: We found that 22 isolates carried the repAci6 gene, suggesting that they contain a RepAci6 plasmid family. Using the primers linking the aphA6 gene to the backbone of repAci6 plasmid, it was revealed that 16 isolates from different hospitals harbored TnaphA6 on a repAci6 plasmid.

Conclusions: This study provides evidence for the dissemination of TnaphA6 on the plasmids encoding RepAci6 in Iranian A. baumannii isolates. Furthermore, it seems that TnaphA6 might be acquired by distinct plasmids separately as it was found to be located on the variants of repAci6 plasmids.
\end{abstract}

Keywords: Acinetobacter baumannii, Amikacin resistance, aphA6, Iran

\section{Background}

Multi-antibiotic resistant (MAR) Acinetobacter baumannii infections are mostly associated with strains belonging to global clone 1 (GC1) and GC2 [1]. Carbapenems have remained the antibiotics of choice for the treatment of infections caused by MAR A. baumannii strains [2]. However, carbapenem resistance has also become widespread in both GC1 and GC2 A. baumannii strains [2]. Aminoglycosides are a potent alternative for the treatment of carbapenem-resistant $A$. baumannii infections [3]. Resistance to aminoglycosides in A. baumannii primarily arises through the genes encoding aminoglycoside-modifying enzymes [4]. Of the genes conferring resistance to aminoglycosides, the aphA6 gene encodes aminoglycoside 3'phosphotransferase. It was first found on a $63-\mathrm{kb}$ plasmid

\footnotetext{
* Correspondence: mdouraghi@tums.ac.ir

${ }^{1}$ Division of Microbiology, Department of Pathobiology, School of Public Health, Tehran University of Medical Sciences, PO Box: 14155-6446, Tehran, Iran

${ }^{3}$ Food Microbiology Research Center, Tehran University of Medical Sciences, Tehran, Iran

Full list of author information is available at the end of the article
}

in a kanamycin-amikacin resistant $A$. baumannii in 1988 . This plasmid, pIP1841, was shown to be transferable to other Acinetobacter species [5]. In Acinetobacter species, the aphA6 gene is commonly found to be flanked by copies of ISAba125 in a composite transposon namely TnaphA6. It is demonstrated that ISAba125 plays a role in overexpression of the aphA6 and high-level resistance to amikacin, kanamycin, and neomycin [6,7]. TnaphA6 was first detected in the Australian isolates belonging to global clone 2 (GC2), then in a member of global clone 1 (GC1) from Australia $[8,9]$, and lately in the isolates containing pCMCVTAb2-type plasmids from the United States [10]. TnaphA6 was also found on pACICU2 in the same location as pAb-G7-2 [11]. These plasmids belong to the most common plasmid family which encodes RepAci6 [9-12]. The plasmids in A. baumannii are categorized into 19 distinct groups on the basis of the nucleotide homology of their respective replicase genes [12]. There are a wide variety of variants of repAci6 plasmids and some have been shown to be conjugative $[6,8,11]$. The 
ubiquitous occurrence of repAci6 plasmid was demonstrated in MAR A. baumannii isolates from 17 European countries [13]. The majority of A. baumannii isolates from European countries (96.8\%) and half of the isolates from Asian countries (51.5 and 46.9\% in Chinese and Taiwanese isolates, respectively) were found to carry repAci6 plasmid [13-15]. The RepAci6 plasmids are important because some of them are associated with the carriage of the resistance genes, including the aphA6, and contribute to the dissemination of the resistance to amikacin $[6,8,11]$. Amikacin resistance due to the TnaphA6 has been shown in the isolates from some countries such as Australia, Italy, and United States [8-11].

In the recent years, a remarkable increase in the resistance to amikacin has been reported - from $58.4 \%$ in 2001-2007 to 95\% in 2012-2013 - in Iran [16], where the aphA6 gene is widespread ranging from 38.8 to $90 \%$ [17-19]. In this study, we examined whether plasmids encoding RepAci6 play a role in the dissemination of the aphA6 gene in GC1 A. baumannii isolates from Iran.

\section{Results}

\section{Phenotype observed}

With respect to phenotypic resistance to amikacin, all the 55 isolates were non-susceptible to amikacin (52 resistant and 3 intermediate susceptible). The antibiotic resistance profile of each strain had been provided through the supplementary data file (see www.karger. com/doi/10.1159/000448785 for all online suppl. material) [20]. Furthermore, the properties of A. baumannii isolates tested are shown in Additional file 1: Table S1.

\section{aphA6 screening}

In total, the aphA6 gene was detected in 54 of the 55 isolates tested. The 52 amikacin-resistant isolates and all but one of the intermediate susceptible isolates harbored the aphA6 gene.

\section{Detecting aphA6 in TnaphA6}

The aphA6 gene is located in TnaphA6 of 52 isolates out of 54 aphA6-harboring isolates as the RH573aphA6R and aphA6F-RH574 PCRs join the aphA6 gene to ISAba125, indicating that the aphA6 is contained within the TnaphA6. The remaining 2 isolates, ABS064 and ABS258, (Additional file 1: Table S1) harbored the aphA6 gene, which was not bound by ISAba125, as the linkage PCRs did not generate amplicons.

\section{Detecting TnaphA6 in Aci6 plasmid backbones}

The screening of the aci6 replication initiation gene revealed that 22 isolates carried the repAci6 gene. Only 16 isolates were found to harbor TnaphA6 on the RepAci6 plasmid. In terms of the presence or absence of PCR amplicons for the different segments of the repAci6 plasmid, these isolates fell into the following groups: i) group 1 included 14 out of 16 isolates from five different hospitals were found to harbor TnaphA6 on a repAci6 plasmid (Table 1). The RH1501-aphA6R and aphA6F-RH1502 linkage PCRs produced amplicons consistent with the predicted amplicon sizes of $1930 \mathrm{bp}$ and $2540 \mathrm{bp}$, respectively. The backbone of repAci6 plasmids is comprised of three segments separated by a repeated sequence. Repeat 1 is $225 \mathrm{bp}$, while repeats 2 and 3 are 422 or $423 \mathrm{bp}$ [9]. Here, we examines if these sequences are present. The PCRs for repeated sequences 1,2 , and 3 produced the expected amplicons, suggesting the presence of a repAci6 plasmid. The sequencing of TnaphA6 besides the repeated sequences 1,2 , and 3 showed that it shares $100 \%$ nucleotide identity to some plasmids such as pAb-G7-2, pCMCVTAb2$\mathrm{Ab} 4, \mathrm{pC} 13-2$, and p1AB5075, which belong to the RepAci6 plasmid family. The complete sequence of TnaphA6 and $4130 \mathrm{bp}$ its flanking sequences were determined from $A$. baumannii strain ABS201 and deposited in the GenBank under the accession number MH500097; ii) the isolate ABI032 (group 2) had a RepAci6 plasmid and generated amplicons consistent with the expected sizes for all the PCRs, except for the repeated sequence 3 . The amplicon size of repeated sequence 3 in this isolate was larger than the expected amplicon size of $1575 \mathrm{bp}$ and was approximately $1800 \mathrm{bp}$. The sequencing of this larger amplicon showed that it shares $99 \%$ nucleotide identity to that of p1AB5075 belongs to the RepAci6 plasmid family [21]; iii) the isolate ABS224 (group 3) also had a RepAci6 plasmid, but did not produce an amplicon for TnaphA6_R, yielded a larger amplicon for repeated sequence 3 and generated all the other amplicons with the expected sizes in PCRs indicated in Table 2. The sequencing of the repeated sequence 3 in addition to repeated sequences 1 and 2 showed that it shares $99 \%$ nucleotide identity to that of pUSA15 belongs to the RepAci6 plasmid family [23]; iv) group 4 included the remaining 6 out of 22 repAci6-carrying isolates, did not produce the amplicons for PCRs linking the aphA6 to the backbone of repAci6 plasmids, TnaphA6_L and TnaphA6_R, and generated a larger amplicon for repeated sequence 3 (Table 1). The 33 isolates did not produce amplicon for the aci6 replicon gene. Thirty out of 33 isolates harbored TnaphA6, but there was no evidence to locate TnaphA6 on plasmids encoding RepAci6 in these isolates. Most of these isolates (29 out of 30) were from the hospital $\mathrm{H} 5$. Of the remaining 3 isolates, 2 carried the aphA6 gene which was not bound by ISAba125 and 1 isolate lacked the aphA6 gene. 
Table 1 Properties of 22 isolates containing repAci6 plasmids

\begin{tabular}{|c|c|c|c|c|c|c|c|c|c|c|c|}
\hline Isolate & $\begin{array}{l}\text { Number of } \\
\text { isolates }\end{array}$ & Year & Hospital & repAci6 & aphA6 & TnaphA6 & $\begin{array}{l}\text { TnaphA6_ } \\
L\end{array}$ & $\begin{array}{l}\text { TnaphA6_ } \\
\text { R }\end{array}$ & $\begin{array}{l}\text { Repeated } \\
\text { sequence } 1\end{array}$ & $\begin{array}{l}\text { Repeated } \\
\text { sequence } 2\end{array}$ & $\begin{array}{l}\text { Repeated } \\
\text { sequence 3 }\end{array}$ \\
\hline Group 1 & & & & + & + & + & + & + & + & + & + \\
\hline ABS103, ABS178, & 14 & $\begin{array}{l}2011- \\
2013\end{array}$ & $\begin{array}{l}\mathrm{H} 1, \mathrm{H} 2, \\
\mathrm{H} 3\end{array}$ & & & & & & & & \\
\hline ABS180, ABS226, & & & $\mathrm{H} 4, \mathrm{H} 5$ & & & & & & & & \\
\hline \multicolumn{12}{|l|}{ ABS290, ABS249, } \\
\hline \multicolumn{12}{|l|}{$\begin{array}{l}\text { ABS288, ABH008, } \\
\text { ABS104, ABS200, }\end{array}$} \\
\hline \multicolumn{12}{|l|}{ ABS201, ABM015, } \\
\hline \multicolumn{12}{|l|}{ ABI031, ABS094 } \\
\hline Group 2 & & & & + & + & + & + & + & + & + & $+^{a}$ \\
\hline ABI032 & 1 & 2012 & $\mathrm{H} 3$ & & & & & & & & \\
\hline Group 3 & & & & + & + & + & + & - & + & + & $+^{\mathrm{a}}$ \\
\hline ABS224 & 1 & 2013 & $\mathrm{H} 5$ & & & & & & & & \\
\hline Group 4 & & & & + & + & + & - & - & + & + & $+^{a}$ \\
\hline ABS101, ABS115, & 6 & 2013 & $\mathrm{H} 5$ & & & & & & & & \\
\hline \multicolumn{12}{|l|}{ ABS138, ABS267, } \\
\hline ABS078, ABS084 & & & & & & & & & & & \\
\hline
\end{tabular}

${ }^{\text {a }}$ The amplicon is larger than the expected size

\section{Discussion}

To date, TnaphA6-carrying plasmids belonging to repAci6 plasmid family have appeared to be mainly restricted to the Australian isolates and have only occasionally been found in the limited number of isolates from Italy [11], Romania [24], and the United States
[10]. The aphA6 has been detected not only in A. baumannii but also on a plasmid of Escherichia coli and Klebsiella pneumoniae isolates from Republic of the Union of Myanmar, Australia, and Italy [25-27]. In addition, this gene was found on a plasmid of Providencia rettgeri isolate from Canada in 2013 [28].

Table 2 The primers used in this study

\begin{tabular}{|c|c|c|c|c|c|}
\hline Target & Primer & Sequence $\left(5^{\prime}-3^{\prime}\right)$ & Annealing temperature $\left({ }^{\circ} \mathrm{C}\right)$ & Amplicon length (bp) & Reference \\
\hline \multirow[t]{2}{*}{ aphA6 } & aphA6F & CATTGCGGGGTTITAATG & 59 & 837 & This study \\
\hline & aphA6R & TTAGATAATGCTTGGAATCA & & & \\
\hline \multirow[t]{2}{*}{ ISAba125-aphA6 } & RH573 & AAGAAGGCTTITCAGCCAGA & 58 & 1427 & [8] \\
\hline & aphA6R & GGACAATCAATAATAGCAAT & & & [22] \\
\hline \multirow[t]{2}{*}{ aphA6-ISAba125 } & aphA6F & ATACAGAGACCACCATACAGT & 58 & 1745 & [22] \\
\hline & RH574 & CAAACATGAGGTGCGACAGT & & & [8] \\
\hline \multirow[t]{2}{*}{ repAci6 } & gr6FW & AGCAAGTACGTGGGACTAAT & 59 & 662 & [12] \\
\hline & gr6RV & AAGCAATGAAACAGGCTAAT & & & [12] \\
\hline \multirow[t]{2}{*}{ TnaphA6_L } & RH1501 & CTTGAGGAAGGGATGGTTGA & 59 & 1930 & [9] \\
\hline & aphA6R & GGACAATCAATAATAGCAAT & & & [22] \\
\hline \multirow[t]{2}{*}{ TnaphA6_R } & aphA6F & ATACAGAGACCACCATACAGT & 59 & 2540 & [22] \\
\hline & RH1502 & TTGCTITAATCGGTGGTTCC & & & [9] \\
\hline \multirow[t]{2}{*}{ Repeated sequence 1} & RH1398 & TTGACGTTGCTCTTGTTGC & 58 & 991 & [9] \\
\hline & RH1399 & TTCTCCCAAGTGGTCAGGTC & & & [9] \\
\hline \multirow[t]{2}{*}{ Repeated sequence 2} & RH1394 & TGGTTGGCAGAACAAGATGA & 58 & 1372 & [9] \\
\hline & RH1395 & TCAAACGATGCAATGGAAGA & & & [9] \\
\hline \multirow[t]{2}{*}{ Repeated sequence 3} & RH1503 & GAAGATCCAGAAGCGGGATA & 58 & 1575 & [9] \\
\hline & RH1397 & CCATGTTCTTTCCACATGC & & & [9] \\
\hline
\end{tabular}


In this study, all isolates harbored aphA6 except one with intermediate susceptibility to amikacin which lacked the aphA6 gene, suggesting that other mechanisms may contribute to the resistance of this isolate to amikacin. Moreover, it was found that the aphA6 in majority of the isolates is located in TnaphA6. This finding is consistent with the previous studies reporting that the aphA6 gene is frequently located in TnaphA6 [8-10].

It was observed that nearly half of the isolates carried repAci6 gene, suggesting that they contain the RepAci6 plasmid family. These isolates fell into the four groups: in group 1, which accounted for three-quarters of the isolates, the positive results of PCRs spanning the aphA6 gene to the backbone of plasmids encoding RepAci6 on the right and left indicate that the TnaphA6 is likely to be present in a repAci6 plasmid family. The only isolate of group 2, had a RepAci6 plasmid and generated amplicons consistent with the expected sizes for all the PCRs, except for the repeated sequence 3 . The amplicon size of repeated sequence 3 in this isolate was larger than the expected amplicon size and may imply that TnaphA6 is located in a different position on the repAci6 plasmid from the isolates that had a usual size for repeated sequence 3 . This finding indicates that TnaphA6 is probably present on a variant of repAci6 plasmid family in this isolate (ABI032). With regard to the isolate in group 3 , our finding probably implies the carriage of TnaphA6 in a different position on a variant of repAci6 plasmid family. The TnaphA6 in the last group of the isolates appears to be probably located on the variants of RepAci6 plasmids or on the other plasmid families or somewhere on the chromosome in these isolates.

Most of the remaining isolates harbored TnaphA6, but there was no evidence to locate TnaphA6 on plasmids encoding RepAci6 in these isolates; therefore, TnaphA6 might be located on the other plasmid families or somewhere on the chromosome.

The limitation of the current study is that all isolates were collected from the hospitals which were located in the same city. Collecting isolates from geographically distant locations in Iran will provide more comprehensive data and conducting such a study can be done in future.

\section{Conclusions}

Using the PCR strategy previously developed [9], this study provides evidence for the dissemination of $\operatorname{Tn} a$ phA6 on the repAci6 plasmids family in amikacinresistant isolates in Tehran, Iran (the second largest country in the Middle East). It appears that these plasmids are widespread in GC1 and 2 strains in the other parts of the world as we detected it in GC1 isolates from different hospitals within Tehran. In addition to the dissemination of TnaphA6 through this plasmid family and the emergence of resistance to aminoglycosides, TnaphA6 might be acquired by each distinct plasmid separately. Further studies are required in order to locate TnaphA6 in other isolates.

\section{Methods}

Bacterial isolates and antimicrobial susceptibility testing

A set of 55 multi-antibiotic resistant $A$. baumannii isolates, which had been previously assigned to GC1 were included in this study [20]. They were collected from 5 hospitals (H1-H5) between 2011 and 2013 in Tehran, Iran. For each strain, at least two overnight cultures in trypticase soy broth (TSB) with $15 \%$ glycerol from the first sub-culture of isolates were stored and then kept at $-70^{\circ} \mathrm{C}$ until further experiments. The identity of $A$. baumannii isolates was confirmed by PCR assays targeting gyrB [29] and/or bla $a_{\text {OxA51-like }}$ [30] genes. In addition to the antibiotic resistance profile determined previously [20], susceptibility testing to amikacin $(30 \mu \mathrm{g})$, kanamycin $(30 \mu \mathrm{g})$, and neomycin $(30 \mu \mathrm{g})$ was performed by disk diffusion method (Mast Diagnostics Ltd., Bootle, Merseyside, $\mathrm{UK}$ ), and the results were interpreted according to the guidelines of the Clinical and Laboratory Standards Institute (CLSI) and the Calibrated Dichotomous Sensitivity (CDS) [31] (http://web.med.unsw.edu/ cdstest). For the strains which were non-susceptible to amikacin by standard disk diffusion method, the minimum inhibitory concentration (MIC) of amikacin (Sigma Aldrich, St Louis, Missouri, USA) was determined. The MIC values were categorized as susceptible (S), intermediate (I), or resistant (R) according to the CLSI guidelines [31]. The protocol followed for disk diffusion and microbroth dilution method is available in the supplementary file.

A. baumannii strain NIPH 10 (provided by Alexander Nemec) was used as a positive control in PCR reaction when screening for the aphA6 gene [3]. As the complete sequence of TnaphA6 in A. baumannii strain ABS201 was deposited in the GenBank (accession number MH500097) during the study period, it was also used as positive control. For negative control in PCR reaction, the DNA template was replaced with DNA of a strain lacking aphA6 gene and sterile distilled water.

\section{Determining the context of aphA6}

PCR was used to examine the presence of the aphA6 gene and also to determine if it is located in TnaphA6. PCR was also used to examine whether TnaphA6 is present in a context similar to the backbone of plasmids encoding RepAci6. Unique sequences on either side of the repeated sequence 1, 2 and 3 (often found in repAci6 plasmids) were joined using previously designed PCRs [9], in cases that TnaphA6 was 
found in a context similar to the backbone of plasmids that carry the repAci6 gene. All primers and their target segments for PCR amplifications are listed in Table 2. The sequencing of the representative PCR amplicons was performed on an Applied Biosystems using the ABI PRISM 3730xl DNA Analyzer (Macrogen Inc., South Korea), and the DNA sequences were analyzed using the online BLAST (http://blast.ncbi. nlm.nih.gov/BLAST.cgi).

\section{Supplementary information}

Supplementary information accompanies this paper at https://doi.org/10. 1186/s12866-019-1592-6.

Additional file 1: Table S1. Properties of Acinetobacter baumannil isolates studied.

\section{Abbreviations}

CDS: Calibrated Dichotomous Sensitivity; CLSI: Clinical and Laboratory Standards Institute; GC1: Global clone 1; GC2: Global clone 2; MAR: Multiantibiotic resistant; MIC: Minimum inhibitory concentration

\section{Acknowledgments}

We thank Dr. Mohammad Hamidian, University of Technology Sydney, Ultimo, NSW, Australia, who spent the countless hours helping us conduct this study.

\section{Authors' contributions}

PA performed the microbiologic and molecular experiments and wrote the manuscript. Besides revising the manuscript, MAB and MD designed, coordinated, and supported this study. All authors have read and approved the version to be published and agreed to be accountable for all aspects of this work.

\section{Funding}

This research was supported by a grant from Tehran University of Medical Sciences \& Health Services (grant number: 37548). The funding body had the role of pay the consumption materials used in this study, and had no role in the design of the study and collection, analysis, and interpretation of data and in writing the manuscript.

\section{Availability of data and materials}

The datasets used and/or analyzed during the current study are available from the corresponding author on reasonable request. All data generated or analyzed during this study are included in this published article and its supplementary information file.

\section{Ethics approval and consent to participate}

The study protocol was in accordance with ethic guidelines of Tehran University of Medical Sciences (TUMS) and the study was approved by the review board at TUMS, Tehran, Iran.

\section{Consent for publication}

Not applicable.

\section{Competing interests}

The authors declare that they have no competing interests.

\section{Author details}

${ }^{1}$ Division of Microbiology, Department of Pathobiology, School of Public Health, Tehran University of Medical Sciences, PO Box: 14155-6446, Tehran, Iran. ${ }^{2}$ Department of Pathology, Tehran Heart Center, Tehran University of Medical Sciences, Tehran, Iran. ${ }^{3}$ Food Microbiology Research Center, Tehran University of Medical Sciences, Tehran, Iran.
Received: 27 November 2018 Accepted: 9 September 2019

Published online: 18 September 2019

\section{References}

1. Diancourt L, Passet V, Nemec A, Dijkshoorn L, Brisse S. The population structure of Acinetobacter baumannii: expanding multiresistant clones from an ancestral susceptible genetic pool. PLoS One. 2010;5(4):e10034.

2. Karageorgopoulos DE, Falagas ME. Current control and treatment of multidrug-resistant Acinetobacter baumannii infections. Lancet Infect Dis. 2008;8(12):751-62

3. Nemec A, Dolzani L, Brisse S, van den Broek P, Dijkshoorn L. Diversity of aminoglycoside-resistance genes and their association with class 1 integrons among strains of pan-European Acinetobacter baumannii clones. J Med Microbiol. 2004;53(12):1233-40.

4. Lambert T, Gerbaud G, Bouvet P, Vieu J, Courvalin P. Dissemination of amikacin resistance gene aphA6 in Acinetobacter spp. Antimicrob Agents Chemother. 1990;34(6):1244-8.

5. Lambert T, Gerbaud G, Courvalin P. Transferable amikacin resistance in Acinetobacter spp. due to a new type of 3'-aminoglycoside phosphotransferase. Antimicrob Agents Chemother. 1988;32(1):15-9.

6. Nigro SJ, Hall RM. Amikacin resistance plasmids in extensively antibioticresistant GC2 Acinetobacter baumannii from two Australian hospitals. J Antimicrob Chemother. 2014;69(12):3435-7.

7. Yoon E-J, Goussard S, Touchon M, Krizova L, Cerqueira G, Murphy C, et al. Origin in Acinetobacter guillouiae and dissemination of the aminoglycosidemodifying enzyme Aph (3')-VI. MBio. 2014;5(5):e01972-14.

8. Nigro SJ, Post V, Hall RM. Aminoglycoside resistance in multiply antibioticresistant Acinetobacter baumannii belonging to global clone 2 from Australian hospitals. J Antimicrob Chemother. 2011;66(7):1504-9.

9. Hamidian M, Holt KE, Pickard D, Dougan G, Hall RM. A GC1 Acinetobacter baumannii isolate carrying $\mathrm{AbaR} 3$ and the aminoglycoside resistance transposon TnaphA6 in a conjugative plasmid. J Antimicrob Chemother. 2013:69(4):955-8

10. Rao J, Susanti D, Childress JC, Mitkos MC, Brima JK, Baffoe-Bonnie AW, et al. Tn2008-driven carbapenem resistance in Acinetobacter baumannii isolates from a period of increased incidence of infections in a Southwest Virginia hospital (USA). J Glob Antimicrob Resist. 2018;12:79-87.

11. Hamidian M, Hall RM. pACICU2 is a conjugative plasmid of Acinetobacter carrying the aminoglycoside resistance transposon TnaphA6. J Antimicrob Chemother. 2013;69(4):1146-8.

12. Bertini A, Poirel L, Mugnier PD, Villa L, Nordmann P, Carattoli A. Characterization and PCR-based replicon typing of resistance plasmids in Acinetobacter baumannii. Antimicrob Agents Chemother. 2010;54(10): 4168-77.

13. Towner K, Evans B, Villa L, Levi K, Hamouda A, Amyes SG, et al. Distribution of intrinsic plasmid replicase genes and their association with carbapenemhydrolyzing class D B-lactamase genes in European clinical isolates of Acinetobacter baumannii. Antimicrob Agents Chemother. 2011:55:2154-9.

14. Chen Y, Gao J, Zhang H, Ying C. Spread of the bla OXA-23-containing Tn2008 in carbapenem-resistant Acinetobacter baumannii isolates grouped in CC92 from China. Front Microbiol. 2017:8:163

15. Liu C-C, Kuo H-Y, Tang CY, Chang K-C, Liou M-L. Prevalence and mapping of a plasmid encoding a type IV secretion system in Acinetobacter baumannii. Genomics. 2014:104(3):215-23.

16. Moradi J, Hashemi FB, Bahador A. Antibiotic resistance of Acinetobacter baumannii in Iran: a systemic review of the published literature. Osong Public Health Res Perspect. 2015;6(2):79-86.

17. Asadollahi K, Taherikalani M, Maleki A, Alizadeh E, Valadbaigi H, Soroush S, et al. Diversity of aminoglycoside modifying enzyme genes among multidrug resistant Acinetobacter baumannii genotypes isolated from nosocomial infections in Tehran hospitals and their association with class 1 integrons. Acta Microbiol Immunol Hung. 2011;58(4):359-70.

18. Farsiani H, Mosavat A, Soleimanpour S, Nasab MN, Salimizand H, Jamehdar SA, et al. Limited genetic diversity and extensive antimicrobial resistance in clinical isolates of Acinetobacter baumannii in north-East Iran. J Med Microbiol. 2015;64(7):767-73

19. Khoshnood S, Eslami G, Hashemi A, Bahramian A, Heidary M, Yousefi N, et al. Distribution of aminoglycoside resistance genes among Acinetobacter baumannii strains isolated from burn patients in Tehran, Iran. Arch Pediatr Infect Dis. 2017;5(3):1-5. 
20. Douraghi M, Jasemi S, Kodori M, Rahbar M, Boroumand MA. Evidence of interruption of the comM gene in a large series of clinical isolates of multidrug-resistant Acinetobacter baumannii. J Mol Microbiol Biotechnol. 2016;26(6):410-3.

21. Gallagher LA, Ramage E, Weiss EJ, Radey M, Hayden HS, Held KG, et al. Resources for genetic and genomic analysis of emerging pathogen Acinetobacter baumannii. J Bacteriol. 2015;197:2027-35.

22. Vila J, Ruiz J, Navia M, Becerril B, Garcia I, Perea S, et al. Spread of amikacin resistance in Acinetobacter baumannii strains isolated in Spain due to an epidemic strain. J Clin Microbiol. 1999:37(3):758-61.

23. Yoon E-J, Kim JO, Yang JW, Kim HS, Lee K, Jeong SH, et al. The bla OXA-23 associated transposons in the genome of Acinetobacter spp. represent an epidemiological situation of the species encountering carbapenems. J Antimicrob Chemother. 2017;72(10):2708-14.

24. Gheorghe I, Novais Â, Grosso F, Rodrigues C, Chifiriuc MC, Lazar V, et al. Snapshot on carbapenemase-producing Pseudomonas aeruginosa and Acinetobacter baumannii in Bucharest hospitals reveals unusual clones and novel genetic surroundings for blaOXA-23. J Antimicrob Chemother. 2015; 70(4):1016-20.

25. Sugawara Y, Akeda Y, Sakamoto N, Takeuchi D, Motooka D, Nakamura S, et al. Genetic characterization of bla $a_{N D M}$-harboring plasmids in carbapenemresistant Escherichia coli from Myanmar. PLoS One. 2017;12(9):e0184720.

26. Espedido BA, Steen JA, Ziochos H, Grimmond SM, Cooper MA, Gosbell IB, et al. Whole genome sequence analysis of the first Australian OXA-48producing outbreak-associated Klebsiella pneumoniae isolates: the resistome and in vivo evolution. PLoS One. 2013:8(3):e59920.

27. Villa L, Capone A, Fortini D, Dolejska M, Rodríguez I, Taglietti F, et al. Reversion to susceptibility of a carbapenem-resistant clinical isolate of Klebsiella pneumoniae producing KPC-3. J Antimicrob Chemother. 2013; 68(11):2482-6.

28. Mataseje L, Boyd D, Lefebvre B, Bryce E, Embree J, Gravel D, et al. Complete sequences of a novel bla NDM-1 $_{1}$-harbouring plasmid from Providencia rettgeri and an Fll-type plasmid from Klebsiella pneumoniae identified in Canada. J Antimicrob Chemother. 2013;69(3):637-42.

29. Higgins $\mathrm{P}$, Wisplinghoff $\mathrm{H}$, Krut O, Seifert H. A PCR-based method to differentiate between Acinetobacter baumannii and Acinetobacter genomic species 13TU. Clin Microbiol Infect. 2007;13(12):1199-201.

30. Woodford N, Ellington MJ, Coelho JM, Turton JF, Ward ME, Brown S, Amyes SG, Livermore DM. Multiplex PCR for genes encoding prevalent OXA carbapenemases in Acinetobacter spp. Int J Antimicrob Agents. 2006;27(4):351-3.

31. Performance Standards for Antimicrobial Susceptibility Testing. 28th ed. CLSI supplement M100. Wayne: Clinical and Laboratory Standards Institute; 2018.

\section{Publisher's Note}

Springer Nature remains neutral with regard to jurisdictional claims in published maps and institutional affiliations.

Ready to submit your research? Choose BMC and benefit from:

- fast, convenient online submission

- thorough peer review by experienced researchers in your field

- rapid publication on acceptance

- support for research data, including large and complex data types

- gold Open Access which fosters wider collaboration and increased citations

- maximum visibility for your research: over $100 \mathrm{M}$ website views per year

At BMC, research is always in progress.

Learn more biomedcentral.com/submissions 